\title{
Mediación comunitaria al cumplimiento del acceso a la justicia como objetivo de desarrollo sostenible ${ }^{1}$
}

\author{
Community mediation for compliance with access to justice as a sustainable development \\ objective
}

Lisbeth Carolina, Velásquez-Cruz ${ }^{2}$

Universidad Nacional Autónoma de Nicaragua, Nicaragua

https://orcid.org/0000-0003-2488-2653

\begin{abstract}
RESUMEN
El presente artículo es producto de una revisión bibliográfica, cuyo objetivo está orientado al análisis de los Objetivos de Desarrollo sostenible, en particular a lo referido a la garantía del acceso a la justicia con igualdad desde los métodos alternos de solución de conflictos, especialmente la mediación comunitaria y la relación con el derecho al acceso a la justicia. Se aplicó el método de análisis documental, con enfoque cualitativo, diseño no experimental, bajo un nivel documental-bibliográfico transversal. Tras la revisión documental se observó que: existen ordenamientos jurídicos nacionales e internacionales y programas en Latino América que regulan la función y practica de la mediación comunitaria como instrumento de solución pacífica de los conflictos en la comunidad. Se concluye que la mediación comunitaria contribuye a crear ciudadanía, participación inclusiva de las personas, desarrollar la cultura de paz en las comunidades y dar cumplimiento al derecho de acceso a la justicia.
\end{abstract}

Palabras claves: acceso a la justicia, desarrollo sostenible, mediación comunitaria, objetivo dieciséis.

\section{ABSTRACT}

This article is the product of a bibliographic review, whose objective is oriented to the analysis of objective sixteen of the Sustainable Development Goals, in particular as regards the guarantee of access to justice with equality from alternative methods of conflict resolution, especially community mediation and the relationship with the right to access justice. The method of documentary analysis was applied, with a qualitative approach, non-experimental design, under a transversal documentary-bibliographic level. After the documentary review, it was observed that: there are national and international legal systems and programs in Latin America that regulate the function and practice of community mediation as an instrument for the peaceful resolution of conflicts in the community. It is concluded that community mediation helps to create citizenship, inclusive participation of people, develop a culture of peace in communities and comply with the right of access to justice.

Keywords: access to justice, community mediation, objective sixteen, sustainable development.

Recibido: 28 de Septiembre 2018 - Aceptado: 20 de Enero 2019

Cómo referenciar este artículo:

Velásquez-Cruz, L. C. (2019). Mediación comunitaria al cumplimiento del acceso a la justicia como objetivo de desarrollo sostenible . Revista Política, Globalidad y Ciudadanía, 134-145. Recuperado de http://revpoliticas. uanl.mx/index.php/RPGyC/article/view/139

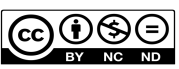

1 Artículo de revisión derivado del proyecto de investigación: Mediación comunitaria como estrategia para desarrollar una cultura de paz en las comunidades.

2 Máster en Derecho Procesal Civil por la Universidad Nacional Autónoma de Nicaragua. Docente de la Facultad de Humanidades y Ciencias Jurídicas de Universidad Nacional Autónoma de Nicaragua. E-mail: lisvelasquezcruz@yahoo.com, lilly121293@hotmail.com.

Revista Política, Globalidad y Ciudadanía, Vol. 5 No. 10, Julio - Diciembre 2019, Universidad Autónoma de Nuevo León, Monterrey, México, ISSN 2395-8448. 134-145. http://revpoliticas.uanl.mx/index.php/RPGyC/article/view/139 


\section{1.- INTRODUCCIÓN}

En el año 2000, los Estados miembros de las Naciones Unidas aprueban la Declaración del Milenio cuyo objetivo primordial fue establecer una serie de Objetivos del Desarrollo del Milenio (ODM) que sean los cimientos indispensables de un mundo mejor, más pacífico, más próspero y justo.

Los Estados partes reconocieron que, "además de las responsabilidades que todos tenemos respecto de nuestras sociedades, nos incumbe la responsabilidad colectiva de respetar y defender los principios de la dignidad humana, la igualdad y la equidad en el plano mundial" (Naciones Unidas, 2000). Esto como parte del cumplimiento efectivo de los Estados a la Carta de Naciones Unidas.

La declaración del milenio contenía ocho objetivos que los Estados miembros definieron de carácter prioritario con relación a las problemáticas que existían en su momento como parte de los compromisos adquiridos en la cumbre:

Reafirmamos nuestra determinación de apoyar todos los esfuerzos encaminados a hacer respetar la igualdad soberana de todos los Estados, el respeto de su integridad territorial e independencia política; la solución de los conflictos por medios pacíficos y en consonancia con los principios de la justicia y del derecho internacional (Naciones Unidas, 2000).

Las problemáticas principales contenidas en los ODM son la erradicación de la pobreza, acceso a educación primaria de forma universal, lucha contra la violencia, protección de Derechos Humanos, la democracia y el buen gobierno, protección de las personas vulnerables, combatir el VIH y Sida y otras enfermedades, reducir la mortalidad de la niñez, medio ambiente sostenible.

Los ODM constituyen "los buenos deseos y al trabajo realizado durante los quince años, los problemas enraizados en muchas sociedades no han sido resueltos y muchas personas en el mundo lo siguen padeciendo como la pobreza extrema" (Quispe Remón, 2018) . Las problemáticas definidas en los objetivos si bien no han sido resueltas en su totalidad constituyen un avance significativo para la creación y readecuación de iniciativas para eliminar las dificultades que generan perjuicios a la sociedad mundial.

Es por lo que, al transcurrir quince años de la Declaración del Milenio, los Estados miembros de las Naciones Unidas se reúnen nuevamente para evaluar el trabajo realizado y establecer las áreas de oportunidad generadas a partir de los ocho objetivos estipulados. Es así como surgen los Objetivos de Desarrollo Sostenible (ODS).

\section{2.- FUNDAMENTO TEÓRICO}

\section{La mirada de los Objetivos de Desarrollo Sostenible}




\section{Objetivos de desarrollo sostenible}

Las Naciones Unidas en aras de continuar en la promoción, protección y cumplimiento de los Derechos y obligaciones señaladas en la Carta de Naciones de Unidas y la Declaración del Milenio en el año 2000, los Estados partes retoman los ODM y los amplían a diecisiete objetivos orientados a la sostenibilidad de estos.

Los objetivos de desarrollo sostenible (ODS) son el sustento y la base para construir un mundo mejor, un marco de referencia en donde los Estados se comprometieron a cumplir, en un máximo de quince años, a partir del año 2015 al 2030. Estableciendo una serie de metas e indicadores que abordaran los avances en la aplicación de estrategias que aportan significativamente a la solución de las problemáticas que aquejan a la sociedad, con el involucramiento de todos los actores claves en el mundo: los Estados, sociedad civil, empresas y población en general.

Los ODS establecidos por las Naciones Unidas son los siguientes:

Ilustración 1 ODS según la ONU

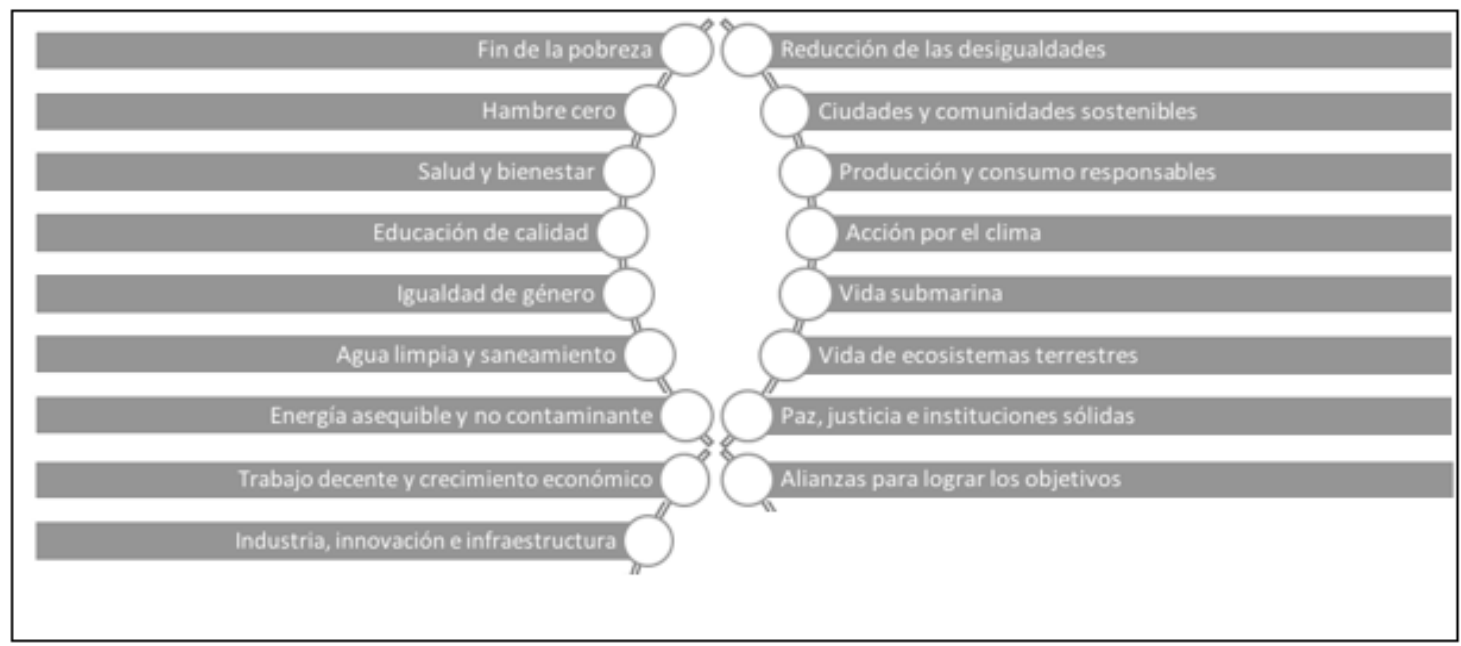

Fuente: Elaboración propia, 2019.

En la ilustración 1, se señalan los objetivos que han sido designados como parte de una nueva agenda de desarrollo sostenible hasta el 2030. En este sentido, Alicia Bárcena Secretaria Ejecutiva de la CEPAL refiere que: "Los ODS también son una herramienta de planificación para los países, tanto a nivel nacional como local. Gracias a su visión a largo plazo, constituirán un apoyo para cada país...”. Es por ello, que los Estados y sus gabinetes de gobiernos preparan y realizan los planes de desarrollo de acuerdo con las principales necesidades y problemáticas que poseen.

Objetivo dieciséis: paz, justicia e instituciones sólidas

Este objetivo propuesto se "centra en la promoción de sociedades pacíficas e inclusivas para

Revista Política, Globalidad y Ciudadanía, Vol. 5 No. 10, Julio - Diciembre 2019, Universidad Autónoma de Nuevo León, Monterrey, México, ISSN 2395-8448. 134-145. http://revpoliticas.uanl.mx/index.php/RPGyC/article/view/139 
el desarrollo sostenible, la provisión de acceso a la justicia para todos y la construcción de instituciones responsables y eficaces a todos los niveles" (CEPAL, 2016). Las tres dimensiones de este objetivo (promoción de la paz, acceso a la justicia e instituciones) se resumen en el fortalecimiento de las instituciones que son garantes de los derechos fundamentales y la vigilancia en el cumplimiento de estos.

Las metas del objetivo dieciséis, según (Naciones Unidas, s.f.) son:

- Reducir significativamente todas las formas de violencia y las correspondientes tasas de mortalidad en todo el mundo.

- Poner fin al maltrato, la explotación, la trata y todas las formas de violencia y tortura contra los niños.

- Promover el estado de derecho en los planos nacional e internacional y garantizar la igualdad de acceso a la justicia para todos

- De aquí a 2030, reducir significativamente las corrientes financieras y de armas ilícitas, fortalecer la recuperación y devolución de los activos robados y luchar contra todas las formas de delincuencia organizada

- Reducir considerablemente la corrupción y el soborno en todas sus formas

- Crear a todos los niveles instituciones eficaces y transparentes que rindan cuentas

- Garantizar la adopción en todos los niveles de decisiones inclusivas, participativas y representativas que respondan a las necesidades

- Ampliar y fortalecer la participación de los países en desarrollo en las instituciones de gobernanza mundial

- De aquí a 2030, proporcionar acceso a una identidad jurídica para todos, en particular mediante el registro de nacimientos

- Garantizar el acceso público a la información y protegerlas libertades fundamentales, de conformidad con las leyes nacionales y los acuerdos internacionales

- Fortalecer las instituciones nacionales pertinentes, incluso mediante la cooperación internacional, para crear a todos los niveles, particularmente en los países en desarrollo, la capacidad de prevenir la violencia y combatir el terrorismo y la delincuencia

- Promover y aplicar leyes y políticas no discriminatorias en favor del desarrollo sostenible

El objetivo dieciséis señala metas para el cumplimiento y logro de este propósito, tomando en cuenta la labor de todos los sectores en la sociedad: gobierno, sociedad civil y población en general. Es importante la integración de todos los sectores para que juntos aporten a la solución y reducción de las problemáticas que acontecen en el mundo. Para (Mesa, 2017, pág. 31) "El ODS 16 ofrece una oportunidad para reflexionar sobre los conceptos de paz, justicia y gobernanza global, así como sobre sus factores constitutivos".

\section{El derecho a la igualdad del acceso a la justicia como objetivo de desarrollo sostenible}

\section{Nociones generales del acceso a la justicia}

Revista Política, Globalidad y Ciudadanía, Vol. 5 No. 10, Julio - Diciembre 2019, Universidad Autónoma de Nuevo León, Monterrey, México, ISSN 2395-8448.134-145. http://revpoliticas.uanl.mx/index.php/RPGyC/article/view/139 
El acceso a la justicia como noción radica en que encierra dos de los propósitos fundamentales del sistema de justicia: por una parte, el acceso al sistema judicial que es donde las personas pueden reclamar el cumplimiento de sus derechos y, por otra, los medios alternos de resolución de conflictos, desde una perspectiva amplia de justicia (judicial y extrajudicial) que involucre a las instituciones públicas en su conjunto.

Tradicionalmente, el acceso a la justicia fue entendido más como una prerrogativa del Estado que como un derecho fundamental y en las últimas décadas ha venido cobrando fuerza la idea del acceso a la justicia como un derecho y más que derecho una garantía fundamental.

En ese aspecto, señala (Marabotto, 2003) "El acceso a la justicia es un derecho humano y, por lo tanto, es esencial. Siempre y en todo caso se debe procurar el acceso a ella de la mayor parte, si no de todos los habitantes de un país". A su vez, la importancia del acceso a la justicia como derecho fundamental es que por medio de este derecho se le da contenido material a la igualdad formal mediante la exigibilidad de otros derechos y la resolución de conflictos entre particulares o entre particulares y el Estado.

Según, (Zambrano, 2015):

El acceso a una justicia imparcial e independiente que garantice la tutela judicial efectiva de los derechos y la vida en un entorno libre de amenazas, violencia y temor, es presupuesto indispensable para alcanzar el desarrollo integral de las personas, mejorar su calidad de vida y lograr el ejercicio pleno de sus derechos y libertades democráticas.

Entonces, el acceso a la Justicia es aquella que no sólo incluye la tutela judicial efectiva y las garantías del debido proceso en el sistema de justicia, sino que abarca todo mecanismo que sea eficaz para la resolución de un conflicto. Por lo mismo, el acceso a la justicia es concebido como una especie de "derecho bisagra", en cuanto permite dar efectividad a los distintos derechos, civiles y políticos y económicos, sociales y culturales, abriendo el camino para reclamar por su cumplimiento y así garantizar la igualdad y la no discriminación.

\section{El acceso a la justicia incluido en el objetivo dieciséis}

El objetivo dieciséis, refiere a la construcción de sociedades justas y pacíficas con el cumplimiento efectivo de los derechos fundamentales para garantizar la paz y la justicia. Los derechos que señala este objetivo es únicamente el de acceso igualitario a la justicia, que, a su vez, este derecho no lo incluía los ODM.

En este sentido, en el centro del objetivo dieciséis se encuentra el aspecto vital para lograr la convivencia pacífica y el desarrollo en igualdad de condiciones de todas las personas, el acceso a la justicia (Mesa, 2017). Es decir, el derecho al acceso a la justicia es transcendente para alcanzar las metas y objetivos señalados en los ODS, ya que a través de este derecho es el medio para el cumplimiento de otros derechos.

\section{Normas de especial protección al derecho del acceso a la justicia}

Revista Política, Globalidad y Ciudadanía, Vol. 5 No. 10, Julio - Diciembre 2019, Universidad Autónoma de Nuevo León, Monterrey, México, ISSN 2395-8448. 134-145. http://revpoliticas.uanl.mx/index.php/RPGyC/article/view/139 
La administración de justicia, como instrumento fundamental para el logro de una convivencia armoniosa y equilibrada, no puede liberarse del compromiso de incorporar en su estructura, organización y políticas, una perspectiva de protección, con el fin de garantizar el acceso a la justicia y con ello, el goce y ejercicio pleno de los derechos humanos y libertades fundamentales, entre ellas vivir libres de cualquier forma de violencia.

El acceso a la justicia se encuentra consagrado en las distintas normas del Derecho Internacional de los Derechos Humanos, como el Artículo 10 de la Declaración Universal de Derechos Humanos, el Artículo 14 del Pacto Internacional de Derechos Civiles y Políticos y el Artículo 8 y 24 de la Convención Americana sobre Derechos Humanos que reconocen al acceso a la justicia como un derecho de que a las personas se les escuchen ante los tribunales y cortes de justicia con igualdad y sin discriminación, que cada Estado debe cumplir con esa garantía fundamental de que las personas puedan libremente tocar las puertas del sistema de justicia y que este les tutele de forma efectiva su derecho.

En este sentido, el acceso a la justicia está consagrado en el artículo 8 de la Convención Americana sobre Derechos Humanos o Pacto de San José, que se encuentra íntimamente ligado con el derecho a la asistencia jurídica, y en general, con poner en funcionamiento el aparato judicial. También se relaciona con la eliminación de todos los impedimentos fácticos, subjetivos u objetivos para hacerlo.

Artículo 8: garantías judiciales (Organización de Estados Americanos, 1969):

Toda persona tiene derecho a ser oída, con las debidas garantías y dentro de un plazo razonable, por un juez o tribunal competente, independiente e imparcial, establecido con anterioridad por la ley, en la sustanciación de cualquier acusación penal formulada contra ella, o para la determinación de sus derechos y obligaciones de orden civil, laboral, fiscal o de cualquier otro carácter.

Esto último se refiere a la posibilidad material del ciudadano y la ciudadana, de acceder a la justicia como elemento que se requiere para poder cumplir con el objetivo que persigue la Ley, como es prevenir, erradicar y sancionar las conductas que ponen en riesgo o vulneran bienes jurídicos protegidos.

\section{La mediación comunitaria y la relación con el acceso a la justicia}

Noción de mediación comunitaria

La mediación comunitaria es parte de los Métodos Alternos de Solución de conflictos, corresponde a la mediación en el ámbito social, según (Nató, Rodríguez, \& Carbajal, 2006):

Es un recurso humano y un instrumento cívico mediante el cual los integrantes de una sociedad pueden tramitar sus diferencias y/o gestionar los conflictos que se le presentan en el ámbito privado y/o público, así como también participar en la construcción de la sociedad que integran.

Para el Programa de Mediación Comunitaria de la Defensoría en Montevideo, Uruguay, "La mediación comunitaria es un espacio de encuentro que busca la construcción de soluciones a las 
situaciones problemáticas que enfrentan las personas" (Defensoría de Vecinas y vecinos, 2017).

En este sentido, la mediación comunitaria es un método de prevención y solución de controversias comunitarias, un servicio que el objetivo primordial es fortalecer la comunidad y generar espacios que involucren a los miembros de la sociedad, a fin de garantizar la paz y armonía entre ellos.

\section{La mediación comunitaria reconocida en normas internacionales}

La Convención Americana sobre Derechos Humanos sirvió de fundamento para abordar los Métodos Alternos de Solución de conflictos, por ende, la mediación comunitaria, en tanto, reconoce las garantías judiciales como el derecho al acceso a la justicia (Organización de Estados Americanos, 1969).

Los Estados signatarios del Tratado Americano de Soluciones Pacíficas mejor conocido como el "Pacto Bogotá", se comprometen a resolver las futuras controversias que pudieran surgir entre ellos, mediante el uso de métodos pacíficos que auspicien el mantenimiento de la paz, como los procedimientos de: los buenos oficios, la mediación, la investigación, la conciliación, el arbitraje y el procedimiento judicial. (Cabello Tijerina \& Vázquez Gutiérrez, 2015).

Siendo la mediación comunitaria parte de los Métodos de solución de conflictos tiene la investidura y protección especial por parte de los Estados que son los garantes de los Derechos, en particular, el derecho al acceso a la justicia. La Administración de Justicia le corresponde al Estado y a su vez, es instrumento fundamental para el logro de una convivencia armoniosa y equilibrada.

\section{Mediación comunitaria y acceso a la Justicia en Nicaragua, México y Panamá}

En Nicaragua, México y Panamá los Mecanismos de Solución de Conflictos en particular la mediación, se encuentran reflejados en los ordenamientos jurídicos desde la perspectiva del derecho humano del acceso a la justicia, la tutela judicial efectiva y el reconocimiento de la participación ciudadana, las formas de gobierno y solución tradicional de conflictos de las comunidades indígenas, zonas urbanas y rurales.

Existen programas, centros y experiencias innovadoras de Mediación comunitaria vinculadas al cumplimiento del derecho al acceso a la justicia de las comunidades, zonas urbanas y rurales, cuyo funcionamiento y operatividad pertenece tanto al Estado (Poder judicial y ejecutivo) como también la sociedad civil y organizaciones internacionales.

Por su parte, la Asamblea Nacional de Panamá dicta la Ley que establece la justicia comunitaria de paz y las disposiciones sobre mediación y conciliación comunitaria, esta norma viene a promover la solución pacifica de las controversias comunitarias a fin de garantizar el acceso a la justicia por igual, sin discriminación de raza, sexo o ideología política, que al igual que la experiencia de Nicaragua crean el Sistema Nacional de Facilitadores Judiciales que junto con

Revista Política, Globalidad y Ciudadanía, Vol. 5 No. 10, Julio - Diciembre 2019, Universidad Autónoma de Nuevo León, Monterrey, México, ISSN 2395-8448. 134-145. http://revpoliticas.uanl.mx/index.php/RPGyC/article/view/139 
el Órgano Judicial y Alcaldías municipales establecen una estructura organizacional en cada municipio.

En el caso de México, específicamente el Estado de Nuevo León incluye los Mecanismos Alternativos de Solución de controversias en el ordenamiento jurídico tomando en consideración la Carta Magna de la Nación y Constitución del Estado Libre y Soberano de Nuevo León que preceptúan el reconocimiento de la mediación para fomentar y difundir la cultura de paz y la restauración de relaciones interpersonales y sociales (Congreso del Estado de Nuevo León, 2017).

En este sentido, el Instituto de Mecanismos Alternativos de Solución de Conflictos del Estado de Nuevo León, ofrece a la sociedad el servicio de Mediación. Según informe actualizado hasta el 26 de marzo de 2019, se realizaron 16,617 mediaciones y 12,593 convenios acordados en total. En el caso de mediaciones comunitarias del 2007 al 30 de abril de 2018 se realizaron 60 solicitudes de mediaciones comunitarias en sede del Instituto (Instituto de Mecanismos Alternativos para la solucion de controversias, s.f.).

En Nicaragua la experiencia de la mediación comunitaria es a través del Servicio Nacional de Facilitadores Judiciales que son auxiliares de justicia, cuenta con 4,764 facilitadores a nivel nacional y se va a seguir ampliando el servicio en 5 mil. El magistrado de la Corte Suprema de Justicia de Nicaragua Dr. Marvin Aguilar expresa que: "El 80\% de la población rural nicaragüense tiene acceso a la justicia a través de los Facilitadores Judiciales.

La eficiencia se percibe en cada comunidad y barrio, con la disminución del delito, lo que genera mayor producción, gobernabilidad, seguridad, paz, armonía, más acceso a la justicia y menos retardo, mayor ejercicio democrático por ser los facilitadores judiciales electos popularmente" (Corte Suprema de Justicia de Nicaragua, 2016).

El Servicio Nacional de Facilitadores Judiciales (SNFJ) tiene como fin brindarle a la comunidad celeridad en el acceso a la justicia, disminución de los costos y se descongestione el sistema de administración de justicia, al no ingresar causa por faltas penales o delitos a la Policía Nacional, Ministerio Público ni a los Despachos Judiciales (Corte Suprema de Justicia, 2015).

También, el acceso a la justicia descansa en las y los líderes comunitarios que con un espíritu voluntario y de servicio, amor a su comunidad, el cual brinda la confianza y proporciona el ambiente adecuado para que las personas que acuden ante él o ella, previo a acuerdos, establezcan la solución de sus conflictos y el facilitador judicial levanta el acta donde se recoge la manifestación de la expresión y voluntad de las partes.

Dentro de las principales logros que ha alcanzado el SNFJ se encuentran la reducción de los conflictos en cada una de las zonas y comunidades del país, facilita la paz y la convivencia social, el cual permite que las personas desprotegidas y con mayores dificultades para acceder a la justicia acudan ante el facilitador judicial para recibir orientación, información o asesoría sobre el conflicto que tenga, se fomenta la cultura jurídica dentro de la población al realizar charlas 
comunitarias el cual es producto de las capacitaciones que reciben por parte del Poder Judicial (Corte Suprema de Justicia de Nicaragua, 2016)

En ese aspecto, han contribuido al desarrollo, gobernabilidad y fortalecimiento democrático y coordinación entre los administradores de justicia e instituciones del Estado, lo cual ha permitido que la administración de justicia sea más efectiva, promoviendo la participación ciudadana e inclusiva.

En resumen, estos países tienen como común denominador el de desarrollar mecanismos que permitan el fácil y oportuno acceso de todas las personas a la justicia, en particular a aquellas de menores ingresos, adoptando medidas que doten de mayor transparencia, eficiencia y eficacia a la labor jurisdiccional. En este contexto, promoverán, desarrollarán e integrarán el uso de métodos alternativos de solución de conflictos en el sistema de justicia (Segunda Cumbre de las Americas, 1998).

\section{3.- MÉTODO}

\section{Diseño}

El enfoque investigativo del presente artículo es cualitativo, de acuerdo con Hernández, Batista y Fernández (2014) "Utiliza la recolección y análisis de los datos para afinar las preguntas de investigación o revelar nuevas interrogantes en el proceso de interpretación” (p.7). Se utilizó la técnica de análisis de contenido, siendo esta "una técnica de investigación para la descripción objetiva, sistemática y cuantitativa del contenido manifiesta de la comunicación” Berel son citado en (Rodríguez-Burgos \& Rivas Castillo, 2018).

El alcance establecido es el exploratorio "emplean cuando el objetivo consiste en examinar un tema poco estudiado o novedoso" (Hernández, Fernández, \& Baptista, 2014). Alcanzando un diseño no experimental "Que se realiza sin la manipulación deliberada de variables y en los que sólo se observan los fenómenos en su ambiente natural para después analizarlos” (Hernández, Fernández, \& Baptista, 2014).

\section{Instrumentos}

Para la construcción del marco teórico-conceptual de la temática "La mediación comunitaria al cumplimiento del acceso a la justicia como objetivo de desarrollo sostenible", se consultaron un total de veintitrés referencias bibliográficas utilizándose como instrumento las ideas, argumentos y proyectos que fueron interpretados desde una perspectiva analítica y crítica.

\section{Procedimiento}

Con relación a la comprensión del problema de la investigación se recopilan fuentes secundarias de documentos académicos. En el marco de referencia se definen los conceptos básicos relativos a los ODS, derecho al acceso a la justicia y la mediación comunitaria. Una vez reco- 
pilada y analizada la información se construye el documento objeto de este trabajo. Por último, se realizan las recomendaciones y conclusiones conforme a los objetivos trazados (Bascón et al, 2016, p. 39).

\section{4.- CONCLUSIONES}

Los objetivos de desarrollo sostenible (ODS) son la base para la construcción de un mundo mejor y el marco de referencia en donde los Estados se comprometieron a cumplir en coordinación con todos los actores claves en la sociedad: gobiernos, sociedad civil y privada y por supuesto la población en general.

El objetivo dieciséis aborda la necesidad de fortalecer instituciones sólidas que fomenten la paz y el cumplimiento efectivo de los derechos sobre todo el de justicia, en el que se promueva el estado de derecho tanto en el ámbito nacional e internacional y garantizar la igualdad de acceso a la justicia para todos.

El acceso a la justicia es un derecho humano y fundamental, es una forma segura de hacer justicia social, de tutelar efectivamente los derechos y garantías de las personas. Es esencial para la construcción de sociedades pacíficas, duraderas y justas.

El acceso a la justicia a través de la mediación comunitaria es una forma novedosa de hacer cumplir este derecho de justicia con igualdad y sin preferencia, facilita la información oportuna para la resolución de un conflicto y no solo eso, sino para aconsejar y realizar actividades que brinde información de diferentes temas y problemáticas que afrente la comunidad. Las personas se sienten en confianza de abordar sus problemas, de acudir ante el facilitador para la solución pacifica de su conflicto.

La mediación comunitaria garantiza el acceso a la justicia en la sociedad y contribuye efectivamente al cumplimiento del objetivo de desarrollo sostenible. En tanto, el acceso a la justicia desde la mediación comunitaria permite el involucramiento y participación inclusiva de la sociedad. La labor de los facilitadores o mediadores comunitarios es a través de la coordinación entre el facilitador como auxiliar de la Administración de justicia entre las distintas Instituciones del Estado y la población, que aporta significativamente al reconocimiento y cumplimiento del derecho al acceso a la justicia y la solución de los conflictos en su comunidad. 


\section{REFERENCIAS}

Bascón, M.: Cazallo, A.; Lechuga, J. y Meñaca, I. (2016). Estudio de la necesidad de implantar un servicio público de transporte entre las ciudades de Ceuta-Tetuán y Melilla-Nador. En Desarrollo Gerencial Revista de la Facultad de Ciencias Económicas, administrativas y contables de la Universidad Simón Bolívar, 8(2), 37 - 57.

Cabello Tijerina, P. A., \& Vázquez Gutiérrez, R. L. (2015). Sistema de solucion de conflictos a la luz del Pacto de Bogotá de 1948. Letras Juridicas.

CEPAL. (2016). Agenda 2030 y los Objetivos de Desarrollo Sostenible. Publicaciones de las Naciones Unidas: una oportunidad para América Latina y el Caribe.

Congreso del Estado de Nuevo León. (2017). Ley de Mecanismos Alternos de Solución Conflictos para el Estado de Nuevo León. Nuevo León, México.

Corte Suprema de Justicia. (2015). Reglamento de Facilitadores Judiciales. Nicaragua.

Corte Suprema de Justicia de Nicaragua. (2016). Impacto del Servicio Nacional de Facilitadores Judiciales. Nicaragua. Obtenido de www.poderjudicial.gob.ni

Defensoría de Vecinas y vecinos. (2017). Mediación comunitaria: Material Didáctico de sensibilización y capacitación. Montevideo, Uruguay: Gliphos.

Hernández, R., Fernández, C., \& Baptista, P. (2014). Metodología de la investigación. México: McGraw-Hill.

Instituto de Mecanismos Alternativos para la solucion de controversias. (s.f.). Instituto de Mecanismos Alternativos para la solucion de controversias. Obtenido de https://www. pjenl.gob.mx/MecanismosAlternativos/

Marabotto, J. (2003). Un derecho humano esencial: el acceso a la justicia. Anuario de Derecho Constitucional Latinoamericano UNAM, 291-301.

Mesa, M. (2017). El ODS 16 sobre paz, seguridad y gobernanza: desafíos conceptuales, seguimiento y evaluación. Centro de educación e investigación para la paz, 31 .

Naciones Unidas . (10 de Diciembre de 1948). Declaración Universal de Derechos Humanos.

Naciones Unidas. (1966). Pacto Internacional de Derechos Civiles y Políticos.

Naciones Unidas. (13 de Septiembre de 2000). Declaración del Milenio. Nueva York.

Naciones Unidas. (s.f.). Naciones Unidas. Obtenido de Naciones Unidas: https://www.un.org/ es/

Nató, A., Rodríguez, M., \& Carbajal, L. (2006). Mediación Comunitaria: Conflictos en el escenario social urbano. Buenos Aires: Universidad.

Organización de Estados Americanos. (1969). Convención Americana sobre Derechos Humanos (Pacto de San José). San José, Costa Rica. 
Quispe Remón, F. (2018). Acceso a la justicia y objetivos del desarrollo sostenible. En D. Verdiales López, Paz, Justicia e instituciones sólidas de derechos humanos y empresas (págs. 235-248). Madrid: Instituto de Estudios Internacionales y Europeos Francisco de Vitoria de la Universidad Carlos III de Madrid.

Segunda Cumbre de las Americas. (1998). Plan de Accion suscrito por los Jefes de Estado y de Gobierno. Chile.

Zambrano, S. (2015). El acceso a la justicia y la tutela judicial efectiva en relacion con la seguridad ciudadana en Ecuador. TLA-MELAUA, revista de Ciencias Sociales, 58-78. 